\title{
Charge Detection in Graphene Quantum Dots
}

\author{
J. Güttinger, C. Stampfer, S. Hellmüller, F. Molitor, T. Ihn and K. Ensslin \\ Solid State Physics Laboratory, ETH Zurich, 8093 Zurich, Switzerland
}

\begin{abstract}
We report measurements on a graphene quantum dot with an integrated graphene charge detector. The quantum dot device consists of a graphene island (diameter $\sim 200 \mathrm{~nm}$ ) connected to source and drain contacts via two narrow graphene constrictions. From Coulomb diamond measurements a charging energy of $4.3 \mathrm{meV}$ is extracted. The charge detector is based on a $45 \mathrm{~nm}$ wide graphene nanoribbon placed approx. $60 \mathrm{~nm}$ from the island. We show that resonances in the nanoribbon can be used to detect individual charging events on the quantum dot. The charging induced potential change on the quantum dot causes a step-like change of the current in the charge detector. The relative change of the current ranges from $10 \%$ up to $60 \%$ for detecting individual charging events.
\end{abstract}

Graphene 1, 2], the two dimensional $\mathrm{sp}^{2}$ allotrope of carbon is a promising material for the development of future nanoelectronics and quantum information processing [3]. This is mainly due to high carrier mobilities [4, 5] and expected long spin lifetimes. Graphene's gapless electronic structure and predicted Klein tunneling through pn-barriers [6] makes it hard to confine charge carriers by electrostatic means. However, by etching graphene it is possible to make tunable graphene nanodevices, as it has been shown by the fabrication of graphene nanoribbons 7, 8, 9], interference devices 10, 11, 12] and graphene quantum dots [13, 14, 15]. In this paper we present an integrated graphene device consisting of a graphene quantum dot with a nearby graphene nanoribbon acting as a quantum-point-contact-like charge detector. Charge detection techniques [16] have been shown to significantly extend the experimental possibilities with quantum dot devices. They are e.g. powerful for detecting spin-qubit states 17, 18 and molecular states in coupled quantum dots [19]. Furthermore charge detectors have been succesfully used to investigate shot noise on a single electron level and full counting statistics [20]. This makes charge detection highly interesting for advanced investigation of graphene quantum dots and graphene nanosystems in general.

Fig. 1a shows a scanning force microscope image of the all graphene structure. The quantum dot device consists of two $35 \mathrm{~nm}$ wide graphene constrictions separating source (S) and drain (D) contacts from the graphene island (diameter $\sim 200 \mathrm{~nm}$ ). The constrictions and the island are electrostatically tuned independently by two barrier gates (B1 and $\mathrm{B} 2$ ) and a plunger gate (PG), respectively. The highly doped silicon back gate (BG) allows to adjust the overall Fermi level. In addition, we placed a $45 \mathrm{~nm}$ wide graphene nanoribbon $60 \mathrm{~nm}$ next to the island, which acts as a charge detector (CD), as shown below.

The sample is fabricated by mechanical exfoliation of natural bulk graphite 21]. Single-layer graphene flakes are transferred to highly doped silicon substrates with a $295 \mathrm{~nm}$ silicon oxide top-layer. Electron beam lithography (EBL) is used for patterning the isolated graphene flakes by subsequent $\mathrm{Ar} / \mathrm{O}_{2}$ reactive ion etching. A second EBL and a lift-off step is performed to place source,

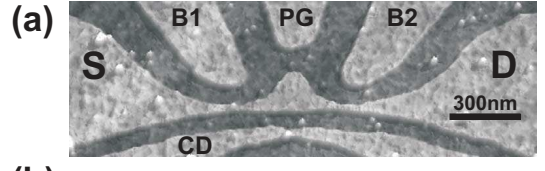

(b)
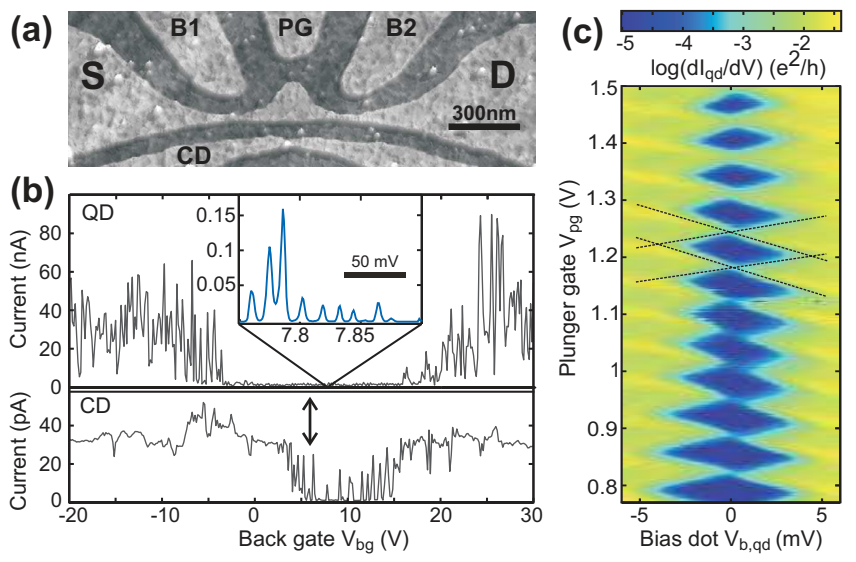

FIG. 1: (Color online) Nanostructured graphene quantum dot device with nanoribbon and characteristic transport measurements. (a) Scanning force micrograph of the measured device. The central island is connected to source (S) and drain (D) contacts by two constrictions. The diameter of the dot is $200 \mathrm{~nm}$ and the constrictions are $35 \mathrm{~nm}$ wide. The graphene nanoribbon acts as charge detector (CD). Three lateral gates $\mathrm{B} 1, \mathrm{~B} 2$ and $\mathrm{PG}$ are used to tune the devices. (b) Back gate characteristics of the quantum dot (QD) device (upper panel) and the charge detector (lower panel), shown in (a). Both measurements were performed at a source-drain (bias) voltage of $V_{b, q d}=V_{b, c d}=500 \mu \mathrm{V}$ and at $1.7 \mathrm{~K}$. Inset shows Coulomb blockade resonances observed inside the transport gap as a function of the back gate voltage over a range of $150 \mathrm{mV}$. (c) Coulomb blockade diamonds in differential conductance (logarithmic scale) recorded as function of the plunger gate and bias voltage with fixed back gate voltage $V_{b g}=2 \mathrm{~V}$. The charging energy is estimated to be $E_{c} \approx 4.3 \mathrm{meV}$.

drain electrodes and contacts to the lateral gate electrodes (all 2/50 nm Ti/Au). For the detailed fabrication process and the single-layer graphene identification by Raman spectroscopy we refer to Refs. [13, 22, 23].

Measurements were performed in a variable temperature insert cryostat at a base temperature of $1.7 \mathrm{~K}$ using low-frequency lock-in techniques.

The characterization of the individual devices is shown in Fig. $1 \mathrm{~b}$ and 1c. Fig. $1 \mathrm{~b}$ shows the current $I$ as a function of the back gate voltage at a temperature of $1.7 \mathrm{~K}$ of both, the quantum dot device (upper curve) and the 


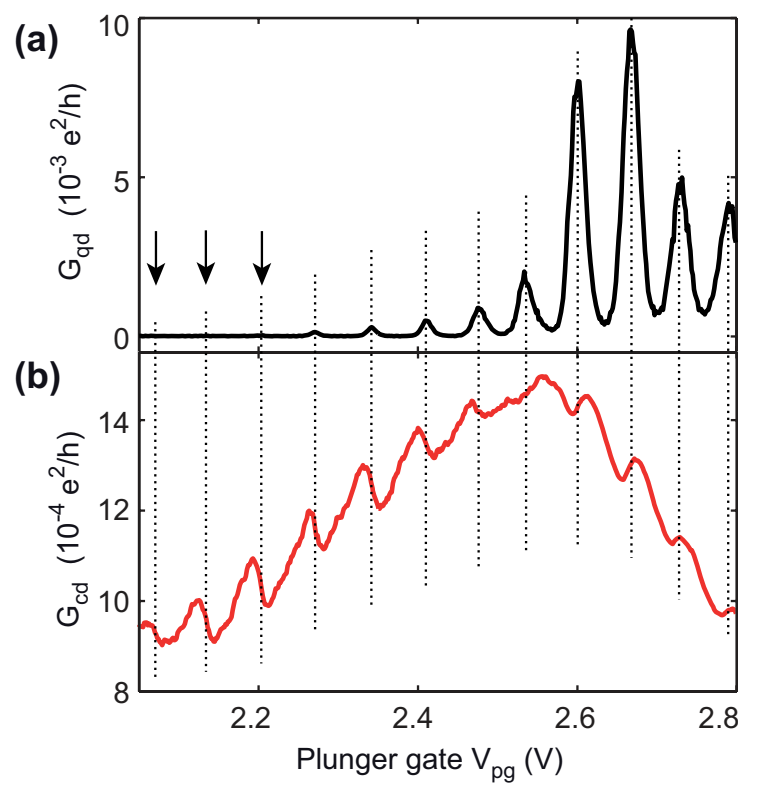

FIG. 2: (Color online). Dot conductance $G_{q d}$ (a) and charge detector conductance $G_{c d}$ (b) as a function of plunger gate voltage $V_{p g}$ for a fixed back gate voltage $V_{b g}=6.5 \mathrm{~V}$. The arrows indicate Coulomb blockade resonances which can be hardly measured by conventional means (a) because the current levels are too low. However, they can be detected by the charge detector (b). Bias on dot: $V_{b, q d}=500 \mu \mathrm{V}$, bias on charge detector: $V_{b, c d}=8.2 \mathrm{mV}$.

charge detector (lower curve). In both cases we observe a transport gap [13] extending roughly from $-4 \mathrm{~V}$ to $15 \mathrm{~V}$ and from $4 \mathrm{~V}$ to $14 \mathrm{~V}$ for the quantum dot, and charge detector, respectively. From high source-drain voltage $\left(V_{b, q d}\right)$ measurements (not shown) we estimate the characteristic energy scale of these effective energy gaps to be about $13 \mathrm{meV}$, and $8 \mathrm{meV}$, respectively. This is in reasonable agreement with recent measurements on graphene nanoribbons where the transport gap is dominated by the width of the graphene nanostructure 24]. The large scale current fluctuations are attributed to resonances in the graphene constrictions. By focusing on a smaller back gate voltage range of $150 \mathrm{mV}$ (see inset in Fig. 1b) Coulomb blockade resonances of the quantum dot are resolved in regions where these resonances are suppressed. In Fig. 1c Coulomb diamond measurements of the quantum dot are shown. The differential conductance of the $\operatorname{dot} d I_{q d} / d V_{b, q d}$ is plotted as a function of the bias voltage $V_{b, q d}$ and plunger gate voltage $V_{p g}$ for a fixed back gate voltage $V_{b g}=2 \mathrm{~V}$. From this measurement a charging energy $E_{c} \approx 4.3 \mathrm{meV}$ and a plunger gate lever arm $\alpha_{p g, q d}=0.06$ is extracted. From further diamond measurements as function of back gate and fixed plunger gate voltage (not shown here) we find a back gate lever arm of $\alpha_{b g, q d}=0.34$.

After having demonstrated the functionality of both devices independently their joint operation is shown in Figs. 2 and 3 where we demonstrate the functionality

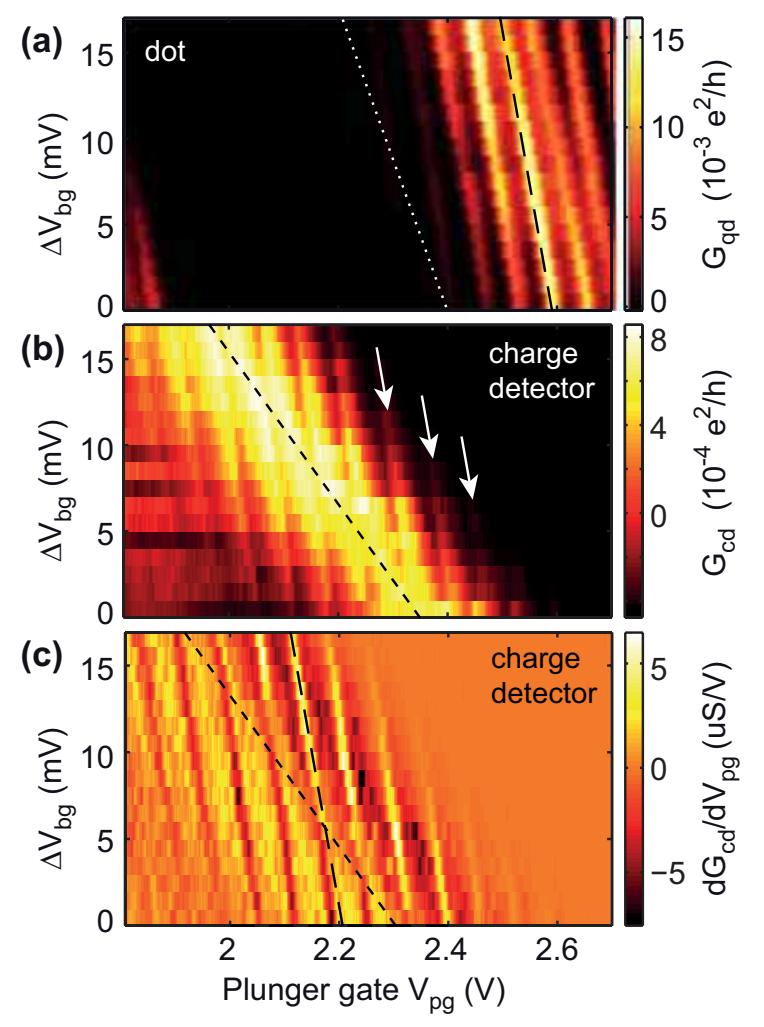

FIG. 3: (Color online). (a) Conductance $G_{q d}$ of the quantum dot as a function of plunger gate voltage $V_{p g}$ and back gate voltage $V_{b g}$. The back gate voltage is converted to a relative scale starting at $V_{b g}=6.505 \mathrm{~V}$ with $\Delta V_{b g}=0$ For this measurement a source drain bias of $V_{b, q d}=500 \mu \mathrm{V}$ is symmetrically applied. Narrowly spaced periodic lines are Coulomb blockade resonances (black line with long dashes), while the larger scale features are attributed to a modulation of the transmission through the barriers (white dotted line). (b) Simultaneous measurement of the charge detector conductance $G_{c d}$. The broad line with increased conductance is less affected by changing the plunger gate voltage compared to the Coulomb blockade resonances in the dot and it is attributed to a local resonance in the charge detector (short dashed line). In addition to this broad line faint lines with a slope corresponding to the Coulomb blockade resonances in the quantum dot are observed (arrows). (c) Derivative of the charge detector conductance plotted in (b) with respect to plunger gate voltage $d G_{c d} / d V_{p g}$, where the lines with short and long dashes indicate the two different lever arms.

and high sensitivity of the graphene charge detector.

For these measurements the back gate voltage is set to $V_{b g}=6.5 \mathrm{~V}$ such that the quantum dot is close to the charge neutrality point (see arrows in Fig. 1b) as well as inside the transport gap of the charge detector. We operate the charge detector in a regime where strong resonances are accessible, in order to make use of steep slopes of the conductance as a function of $V_{p g}$ of the order of 4-6 $10^{-4}\left(e^{2} / h\right) / 100 \mathrm{mV}$ to detect individual charging events on the quantum dot.

Fig. 2a shows almost equidistantly spaced $\left(\Delta V_{p g}=\right.$ 
$65 \pm 3.8 \mathrm{mV}$ ) Coulomb blockade resonances as function of $V_{p g}$ at $V_{b, q d}=500 \mu \mathrm{V}$. The strong modulation of the conductance peak amplitudes is due to superimposed resonances in the graphene constrictions defining the island [13]. In Fig. 2b we plot the simultaneously measured conductance through the nearby charge detector (at a bias voltage of $V_{b, c d}=8.2 \mathrm{mV}$ ) for the same $V_{p g}$ range. On top of the peak-shaped CD resonance we observe conductance steps which are well aligned (see dotted lines) with single charging events on the nearby quantum dot.

Figs. $3 \mathrm{a}$ and $3 \mathrm{~b}$ show $2 \mathrm{D}$ plots of a set of traces corresponding to those shown in Figs. 2a and 2b, taken for different back gate voltages and $V_{b, c d}=V_{b, q d}=500 \mu \mathrm{V}$. Fig. 5a shows Coulomb blockade resonances in the quantum dot conductance following a relativ lever arm of PG and BG of $\alpha_{p g, q d} / \alpha_{b g, q d}=0.18$ (see black dashed line). In Fig. 3b these resonances are observed through charge detection and are marked with arrows. The charge detector resonance used for detection can be distinguished from the dot resonances by its larger width and its different slope given by $\alpha_{p g, c d} / \alpha_{b g, c d}=0.04$ (black dashed line). This reduced slope is due to the larger distance of the CD nanoribbon to the plunger gate $(\sim 350 \mathrm{~nm})$ as compared to the island-plunger gate distance. The modulation of the Coulomb blockade resonances in Fig. 3a is due to resonances in the tunneling constriction located around $300 \mathrm{~nm}$ away from the PG (see Fig. 1a). This leads to a slope of 0.08 for these peak modulations (see white dotted line). Independent of this modulation we identify single charging events on the quantum dot as conductance fringes (see arrows in Fig. 3b) on top of the up and down slope of the CD resonance. This can even better be seen by numerical differentiation of $G_{c d}$ vs. $V_{p g}$, as shown in Fig. 3c. Here the sharp conductance changes due to the charging events in the dot are strongly pronounced, and both relative lever arms to the Coulomb blockade peaks and the constriction resonance in the charge detector are indicated by dashed lines. The detection range can be improved by increasing the bias $V_{b, c d}$, leading to a broadening of the constriction resonance, as seen by comparing Fig. 3c with Fig. 2b.

From the measurement shown in Fig. 2b a nanoribbon conductance change of up to $10 \%$ can be exctracted for a single charging event. For lower bias voltages (e.g. $\left.V_{b, c d}=500 \mu \mathrm{V}\right)$ the change in the conductance can be incresed to $60 \%$.

In conclusion, we demonstrated the functionality of an integrated graphene charge detector based on a nanoribbon nearby a graphene quantum dot. We confirm the detection of charging events in regimes where Coulomb blockade resonances can hardly be measured (i.e. resolved) because the current levels are too low (see, e.g., arrows in Fig. 2a). In contrast to state-of-the-art quantum point contact charge detectors we do not make use of slopes to quantized conductance plateaus. We rather use local resonances in the graphene nanoribbon to detect charging. We anticipate, that this technique will play an important role for the investigation of the electronhole crossover and spin-states in graphene quantum dots.

The authors wish to thank R. Leturcq, P. Studerus, C. Barengo, P. Strasser, A. Castro-Neto and K. S. Novoselov for helpful discussions. Support by the ETH FIRST Lab, the Swiss National Science Foundation and NCCR nanoscience are gratefully acknowledged.
[1] A. K. Geim and K. S. Novoselov, Nat. Mater. 6, 18 (2007).

[2] M. I. Katsnelson, Mater. Today 10, 20 (2007).

[3] B. Trauzettel, D.V. Bulaev, D. Loss, and G. Burkard, Nature Physics, 3, 192, (2007).

[4] K. S. Novoselov, A. K. Geim, S. V. Morozov, D. Jiang, M. I. Katsnelson, I. V. Grigorieva, S. V. Dubonos, and A. A. Firsov, Nature, 438, 197-200, (2005).

[5] Y. Zhang, Y.-W. Tan, H. L. Stormer, and P. Kim, Nature, 438, 201-204, (2005).

[6] M. I. Katsnelson, K. S. Novoselov, amd A. K. Geim, Nature Phys. 2, 620625 (2006).

[7] Z. Chen, Y. Lin, M. Rooks, and P. Avouris, Physica E, 40, 228, (2007).

[8] M. Y. Han, B. Özyilmaz, Y. Zhang, and P. Kim, Phys. Rev. Lett., 98, 206805 (2007).

[9] X. Li, X. Wang, L. Zhang, S. Lee, and H. Dai, Science, 319, 1229 (2008).

[10] F. Miao, S. Wijeratne, Y. Zhang, U. C. Coskun, W. Bao, and C. N. Lau, Science, 317, 1530 (2007).

[11] S. Russo, J. B. Oostinga, D. Wehenkel, H. B. Heersche, S. S. Sobhani, L. M. K. Vandersypen, and A. F. Morpurgo, Phys. Rev. B, 77, 085413 (2008).
[12] F. Molitor, A. Jacobson et.al., to be published (2008)

[13] C. Stampfer, J. Güttinger, F. Molitor, D. Graf, T. Ihn, and K. Ensslin, Appl. Phys. Lett., 92, 012102 (2008).

[14] C. Stampfer, E. Schurtenberger, F. Molitor, J. Gttinger, T. Ihn, and K. Ensslin, Nano Lett. 8(8), 2378 (2008).

[15] L. A. Ponomarenko, F. Schedin, M. I. Katsnelson, R. Yang, E. H. Hill, K. S. Novoselov, and A. K. Geim, Science, 320, 356 (2008).

[16] M. Field, C. G. Smith, M. Pepper, D. A. Ritchie, J. E. F. Frost, G. A. C. Jones, and D. G. Hasko, Phys. Rev. Lett. 70, 1311 (1993).

[17] J. R. Petta, A. C. Johnson, J. M. Taylor, E. A. Laird, A. Yacoby, M. D. Lukin, C. M. Marcus, M. P. Hanson, and A. C. Gossard, Science, 309, 2180-2184 (2005).

[18] J. M. Elzerman, R. Hanson, L. H. Willems van Beveren, B. Witkamp, L. M. K. Vandersypen, and L. P. Kouwenhoven, Nature 430, 431 (2004).

[19] L. DiCarlo, H. Lynch, A. Johnson, L. Childress, K. Crockett, C. Marcus, M. Hanson, and A. Gossard, Phys. Rev. Lett. 92, 226801 (2004) .

[20] S. Gustavsson, R. Leturcq, B. Simovic, R. Schleser, T. Ihn, P. Studerus, and K. Ensslin, Phys. Rev. Lett. 96, 076605 (2006). 
[21] K. S. Novoselov, A. K. Geim, S. V. Morozov, D. Jiang, M. I. Katsnelson, S. V. Dubonos, I. V. Grigorieva, and A. A. Firsov, Science, 306, 666, (2004).

[22] A. C. Ferrari, J. C. Meyer, V. Scardaci, C. Casiraghi, M. Lazzeri, F. Mauri, S. Piscanec, D. Jiang, K. S. Novoselov, S. Roth, and A. K. Geim, Phys. Rev. Lett. 97, 187401
(2006).

[23] D. Graf, F. Molitor, K. Ensslin, C. Stampfer, A. Jungen, C. Hierold, and L. Wirtz, Nano Lett., 7, 238 (2007).

[24] F. Sols, F. Guinea, and A. H. Castro Neto, Phys. Rev. Lett., 99, 166803 (2007). 